\section{EMMPRIN and survivin: prognostic factors in cisplatin-treated patients with bladder cancer}

For patients with advanced bladder cancer, cisplatin-containing chemotherapy is the only treatment option. Despite the existence of various clinical prognostic factors that correlate with the treatment outcome in these patients, no prognostic biological markers are currently used in clinical practice. Als and coauthors identified two new molecular markers prognostic for survival in patients with bladder cancer, by use of gene-expression profiling.

The study included samples from 30 patients with locally advanced and/or metastatic bladder cancer who had received cisplatin-containing chemotherapy. Analysis of microarray data identified 55 differentially expressed genes that correlated significantly with survival after chemotherapy. Multivariate analysis showed that protein expression of EMMPRIN (BSG) and survivin (BIRC5) independently predicted poor outcome (hazard ratio [HR] 2.23, $P<0.0001$, and HR 2.46, $P<0.0001$, respectively) as did the presence of visceral metastases (HR 2.62; $P<0.0001)$. Patients without visceral metastases who had tumors with positive expression of both EMMPRIN and survivin had a low median survival time. The 5-year survival rates of patients without visceral metastases whose tumors expressed none, one or both proteins were $44 \%, 21 \%$ and $0 \%$, respectively. The response rates in patients with or without EMMPRIN expression were $39 \%$ and $74 \%$, respectively, while the response rates in patients with or without survivin expression were $47 \%$ and $70 \%$, respectively.

This study shows that expression of EMMPRIN and survivin can be used as prognostic factors for treatment response and survival in cisplatin-treated patients with advanced bladder cancer.

Original article Als AB et al. (2007) Emmprin and survivin predict response and survival following cisplatin-containing chemotherapy in patients with advanced bladder cancer. Clin Cancer Res 13: 4407-4414

\section{Combined RFA and ethanol injection produces better outcomes than RFA alone in HCC}

Local ablation for hepatocellular carcinoma $(\mathrm{HCC})$ is recommended for patients who cannot undergo lesion resection and as a palliative treatment before liver transplantation. Radiofrequency ablation (RFA) and percutaneous ethanol injection (PEI) are two ablative therapies that have been widely used in patients with HCC; however, the complete tumor necrosis rate achieved with these therapies in tumors larger than $5 \mathrm{~cm}$ is unsatisfactory. To test the hypothesis that the combined use of RFA and PEI might result in greater patient survival than the use of RFA alone, Zhang and colleagues conducted a prospective randomized trial.

In this trial, 133 patients were randomized to receive RFA and PEI ( $n=66 ; 86 \%$ male), or RFA alone ( $n=67 ; 87 \%$ male). Overall survival rates for the RFA + PEl group were significantly higher than those for the RFA-only group at both 3 and 5 years $(P=0.01$ and $P=0.03$, respectively). Stratification by tumor size revealed that patients with $3.1-5.0 \mathrm{~cm}$ tumors had a significantly higher 3-year overall survival rate when treated with RFA and PEI than when treated with RFA alone $(P=0.04)$; rates of 3-year overall survival did not differ between the two treatment groups in patients with tumors $5.1-7 \mathrm{~cm}$ or $<3.0 \mathrm{~cm}$ in size. Local recurrence was significantly lower in the RFA+PEI group than in the RFA-alone group $(P=0.01)$.

Taken together, these results indicate that a treatment regimen that combines RFA and PEI produces better local tumor control and long-term survival in HCC than does RFA alone.

Original article Zhang YJ et al. (2007) Hepatocellular carcinoma treated with radiofrequency ablation with or without ethanol injection: a prospective randomized trial. Radiology 244: 599-607

\section{Two-week-wait rule for breast cancer is ineffective in the UK}

In the UK, high breast-cancer mortality and long waiting lists resulted in release of a 1998 Department of Health directive, followed by a National Health Service Cancer Plan, aimed at addressing these issues. The guidelines stipulated that patients with suspected breast cancer should be seen by a specialist within 2 weeks of referral by a general practitioner. New data indicate that this system is failing, increasing the number of cancer diagnoses in patients referred via the 'routine' non-urgent route and lengthening the time to diagnosis in many. 